\title{
A Histopathological Study of Skin Biopsy Specimens in a Tertiary Care Hospital with a Keynote on Clinicopathological Correlation
}

\author{
Varughese Padinjattadathu George ${ }^{1}$, Sowmya $S^{1 *}$, Krishnan $\mathrm{S}^{2}$ \\ 'Department of Pathology, Mahatma Gandhi Medical College and Research Institute, Sri Balaji Vidyapeeth University, Puducherry, India \\ ${ }^{2}$ Department of Dermatology, Venereology and Leprology, Mahatma Gandhi Medical College and Research Institute, \\ Sri Balaji Vidyapeeth University, Puducherry, India
}

\begin{abstract}
Introduction: The prevalence of dermatological lesions is notably high among tropical countries and the spectrum varies significantly with geographical distribution and co-existing disorders leading to many differential diagnoses. A histopathological examination is essential to arrive at a final diagnosis, thus paving the way for clinicopathological correlation.

Materials and Methods: This is an observational study overa period of 10 years in the Department of Pathology at a rural area based tertiary care hospital. A histopathological analysis of 800 skin biopsy specimens was carried out and slides were stained with Hematoxylin and Eosin stains. Special stains such as Fite-Faraco and Ziehl-Neelsen stains and immunohistochemical stains such as S100 were applied in warranted cases. The data obtained were tabulated and analyzed for clinicopathological correlation.

Results: Among 800 cases analyzed, specific histopathological diagnosis was made in $87.9 \%$ of cases. Neoplastic skin lesions were seen in $22.4 \%$ of cases, whereas $77.6 \%$ of cases were non-neoplastic. Leprosy was found to be the most common skin lesion. Clinicopathological correlation showed concordance in 430 cases (54\%) with significant correlation in leprosy and malignant melanocytic skin tumors.

Conclusion: Granulomatous inflammation is still rampant with leprosy and tuberculosis as the leading causes in spite of extensive programmes and preventive measures. In cases where skin biopsy delivered a non-specific diagnosis, infective or neoplastic etiology was ruled out. The heterogeneity in the clinical presentation of skin diseases makes histopathological examination a gold standard tool to establish both the final diagnosis and clinicopathological correlation.
\end{abstract}

Keywords: Dermatological lesions,Skin biopsies, Histopathological examination, Clinicopathological correlation.

\section{Introduction}

Dermatological disorders are one among the frequently encountered health disorders in tropical countries such as India with varied spectrum depending on geographical domicile. The spectrum of cutaneous disorders varies in their severity ranging from benign forms to life-threatening lesions. ${ }^{1}$ Various factors contribute to this high incidence which includes environmental factors, social customs, economy and literacy. ${ }^{2}$

In spite of common occurrence of the dermatological conditions, they are usually assumed to be insignificant due to the assumption that skin diseases are benign and not life-threatening by nature. ${ }^{3}$ The spectrum of cutaneous disorders is highly variable and hence the standalone clinical diagnosis is often difficult for different entities. Though the anatomical location, distribution, and the pattern of skin lesions help the clinician to diagnose the disease, the histopathological study of the lesion remains as the gold standard method for confirming the diagnosis. ${ }^{4}$

Clinicopathological correlation aids in arriving at an accurate diagnosis in cases with atypical presentation and to plan appropriate treatment modality especially in rare entities and in conditions where histopathology features vary from the clinical impression. ${ }^{5,6}$ Only a few research studies have been carried out in the eastern coastal region which highlights a wide range of skin disorders that require histological examination to aid in clinical diagnosis. Among the available literature, only the statistical analysis is studied, whereas the emphasis on the spectrum and clinicopathological correlation is very sparse. ${ }^{7}$

This study aims at evaluating the histopathological spectrum of skin lesions and to analyze the clinicopathological correlation in various categories of skin lesions.

\section{Material and Methods}

This cross-sectional study was conducted in the Department of Pathology with clearance from the Institutional Ethics Committee during a time period of 10 years from January 2008 to April 2018. During this period, 800 skin biopsies received from the Department of Dermatology, Venereology and Leprology were studied and analyzed. The identities of patients were double-blinded to avoid bias. The blocks/slides were alphabetically coded and 
numbered for the purpose of the study. Archived blocks/ slides and records were accessed from the archives of the hospital and the Department of Pathology for retrospective cases and studied. For prospective cases, the specimens received from November 2016 till April 2018 were studied.

The formalin-fixed tissue bits were subjected to paraffin processing and the paraffin-embedded sections were routinely stained with Hematoxylin and Eosin . Special stains such as Ziehl-Neelsen stain for cutaneous tuberculosis and leprosy, Fite-Faraco for leprosy, Periodic acid-Schiff for fungal infections and adnexal tumors, Masson's Trichome stain for soft tissue tumors and immunohistochemical markers such as S100 for malignant melanoma were added as required for diagnostic purposes.

The clinical parameters were analyzed and skin lesions were histopathologically classified into non-neoplastic and neoplastic lesions. Non-neoplastic skin lesions were further classified into inflammatory lesions comprising of a spectrum of infectious, granulomatous, papulosquamous, vesiculobullous skin lesions, genodermatosis, non-specific dermatitis and miscellaneous skin lesions. Neoplastic skin lesions were further classified into benign and malignant entities. The clinicopathological correlation was performed in feasible cases in reference to standard literature. The data obtained was entered in Microsoft Excel sheet, analyzed using SSPS version 22 software and a descriptive statistical analysis was done. The results were expressed in frequencies, percentages, mean and standard deviation. Chi-square test was used for categorical variables. p-value $<0.05$ was considered significant.

\section{Results}

Among 800 biopsy specimens analyzed, a variety of skin lesions were seen across all age groups and in both genders (Figure 1). The age group of 21-30 years constituted $18.6 \%$ of the total cases. There was a male preponderance (51\%). A specific diagnosis was obtained in 703 cases $(87.9 \%)$ on histopathological examination, whereas a specific histopathological diagnosis could not be made out in 97 cases $(12.1 \%)$,. Inflammatory lesions were the most common lesions ( 476 cases, $59.5 \%$ ) followed by neoplastic lesions (179 cases, 22.4\%) (Table 1). Out of the 476 inflammatory lesions, 121 cases $(15.1 \%)$ had infectious etiology, whereas 355 cases (44.3\%) were non-infectious. Inflammatory lesions were classified as granulomatous (120 cases, 15\%) and non- granulomatous (356 cases, 44.5\%). Inflammatory lesions also included non-infectious papulosquamous lesions (118 cases, 14.8\%), connective tissue disorders (44 cases, 5.5\%), vasculitis (53 cases, $6.6 \%$ ) and vesciculobullous lesions (38 cases, $4.8 \%$ ).
Among the 121 infectious skin lesions, bacterial etiology was found in 98 cases followed by viral etiology in 17 cases, fungal etiology in 5 cases and a solitary case with parasitic etiology. Among the granulomatous skin lesions, 77 cases of leprosy were observed, followed by 23 cases of cutaneous tuberculosis, 5 cases each of granuloma annulare and fungal infections; 2 cases each of foreign body granuloma and chronic granulomatous disease, 3 cases of xanthogranulomas, 1 case each of necrobiosis lipoidica, parasitic infection and cutaneous sarcoidosis. $22.5 \%$ of the granulomatous skin lesions were seen in the age group of 21 to 30 years. Granulomatous skin lesions were seen in $62.5 \%$ of males and $37.5 \%$ of females. Among leprosy cases, borderline tuberculoid type constituted 34 cases followed by lepromatous leprosy in 17 cases, 9 cases of tuberculoid leprosy, 6 cases of mid-borderline leprosy cases, 8 cases of borderline lepromatous, 2 cases of indeterminate leprosy and 1 case of histoid leprosy.

Among non-infectious papulosquamous lesions, lichen planus constituted 43 cases (36.4\%) of lesions followed by 34 cases of psoariasis, 17 cases of pityriasis rubra pilaris, 10 cases of pityriasis rosea, 8 cases of pityriasis lichenoid, 4 cases of lichen nitidus and 2 cases of parapsoriasis. Among the connective tissue disorders, morphea constituted 31 cases and lupus erythematosis was found in 13 cases. Among vasculitis, 51 cases were leucocytoclastic vasculitis, whereas only 1 case of Henoch-Schonlein purpura was reported. Among vesicobullous lesions, 13 cases each of pemphigus vulgaris and bullous pemphigoid were studied followed by 4 cases each of pemphigus foliaceus and dermatitis herpetiformis.

Out of 192 cases of neoplastic skin lesions, 122 cases were benign and the rest 70 cases proved to be malignant. Among the benign skin lesions, keratinocytic tumors constituted 35 cases, followed by vascular tumors (19 cases) and melanocytic tumors (18 cases). Keratinocytic tumors were also the most frequently encountered malignant skin lesions (43 cases) followed by melanocytic tumors (15 cases).

Among the 800 specimens studied, clinicopathological correlation was found in $54 \%$ of cases. The clinicopathological correlation of various classes of skin lesions were analyzed (Table 2). Infectious skin lesions with bacterial etiology showed a clinicopathological correlation of $94.9 \%$. Among infectious granulomatous skin lesions, leprosy (Figures 2 and 3) showed a 100\% clinicopathological correlation. Among non-infectious papulosquamous skin lesions, psoriasis showed 85.3\% clinicopathological correlation, whereas lesions such as pityriasis lichenoid showed no clinicopathological correlation at all. $90.3 \%$ clinicopathological correlation 
was observed in morphea among connective tissue disorders, whereas only $30.8 \%$ of skin lesions with lupus erythematosus showed clinicopathological correlation.

Leucocytoclastic vasculitis showed very good clinicopathological correlation of $82.7 \%$ among cutaneous vasculitis. 28 cases $(73.7 \%)$ of vesiculobullous diseases (Figure 4) had clinicopathological correlation. Omit this sentence.

Table 1: Histopathological spectrum of skin lesions.

\begin{tabular}{|c|c|}
\hline Type of skin lesion & Number of lesions (in percentage) \\
\hline Inflammatory & $476(59.5 \%)$ \\
\hline Neoplastic & $179(22.4 \%)$ \\
\hline Genodermatosis & $21(2.6 \%)$ \\
\hline Miscellaneous & $27(3.4 \%)$ \\
\hline Non-specific & $97(12.1 \%)$ \\
\hline Total & $\mathbf{8 0 0 ( 1 0 0 \% )}$ \\
\hline
\end{tabular}

Table 2: Clinicopathological correlation of various classes of skin lesions.

\begin{tabular}{|c|c|}
\hline Skin Lesions & Clinicopathological Correlation (in percentage) \\
\hline Infectious skin lesions & $86.8 \%$ \\
\hline Cutaneous vasculitis & $83.0 \%$ \\
\hline Granulomatous skin lesions & $80.0 \%$ \\
\hline Vesiculobullous skin lesions & $73.7 \%$ \\
\hline Connective skin disorders & $72.7 \%$ \\
\hline Non-infectious papulosquamous skin lesions & $65.2 \%$ \\
\hline Malignant skin tumors & $58.6 \%$ \\
\hline Benign skin tumors & $47.5 \%$ \\
\hline
\end{tabular}

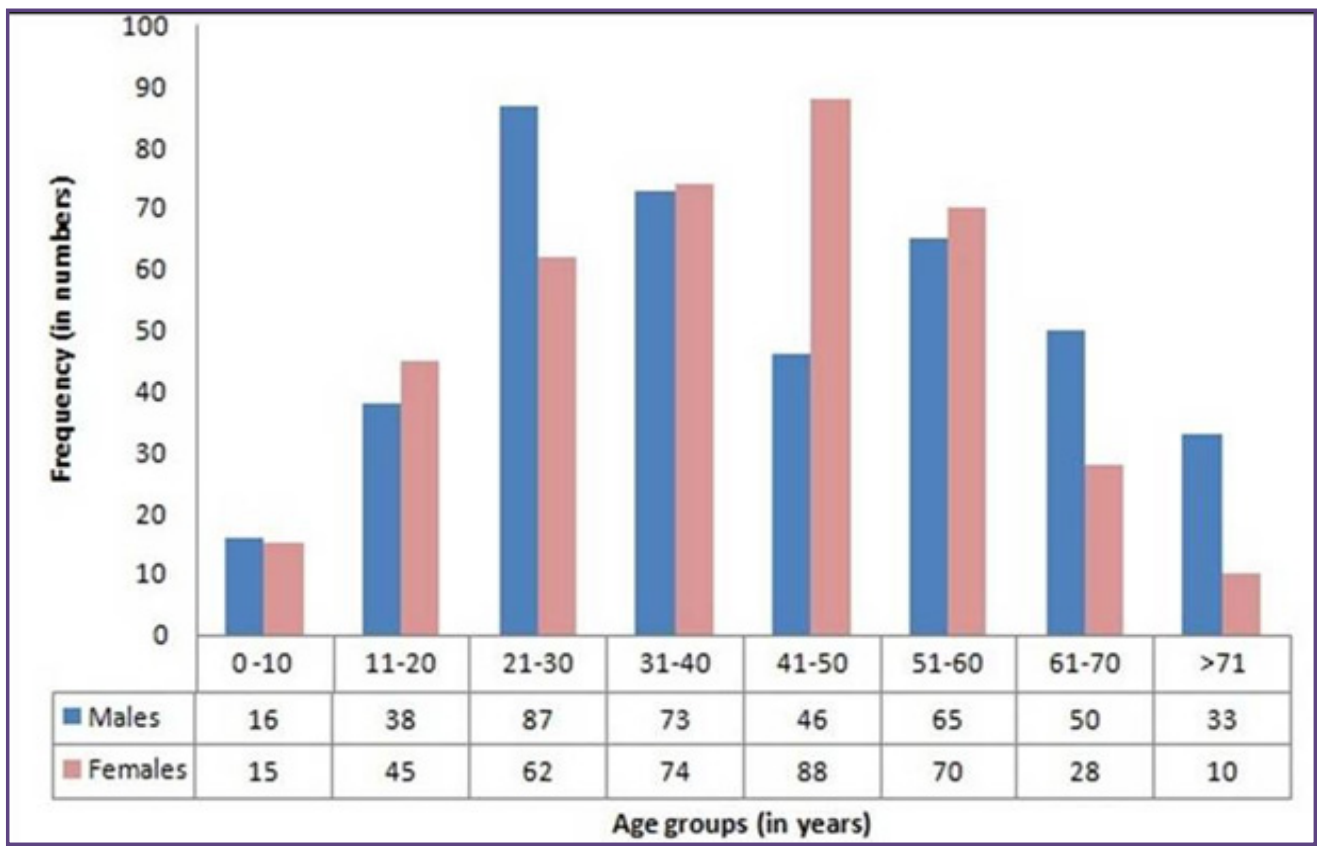

Fig. 1: Age and gender distribution of skin lesions. of $47.5 \%$; among which $83.3 \%$ of melanocytic tumors correlated well with clinical impression, whereas only $13.3 \%$ of tumors with follicular differentiation showed clinicopathological correlation. Among malignant tumors, melanocytic tumors (Figure 5) had 100\% clinicopathological correlation, whereas tumors with follicular differentiation showed nil clinicopathological correlation. 


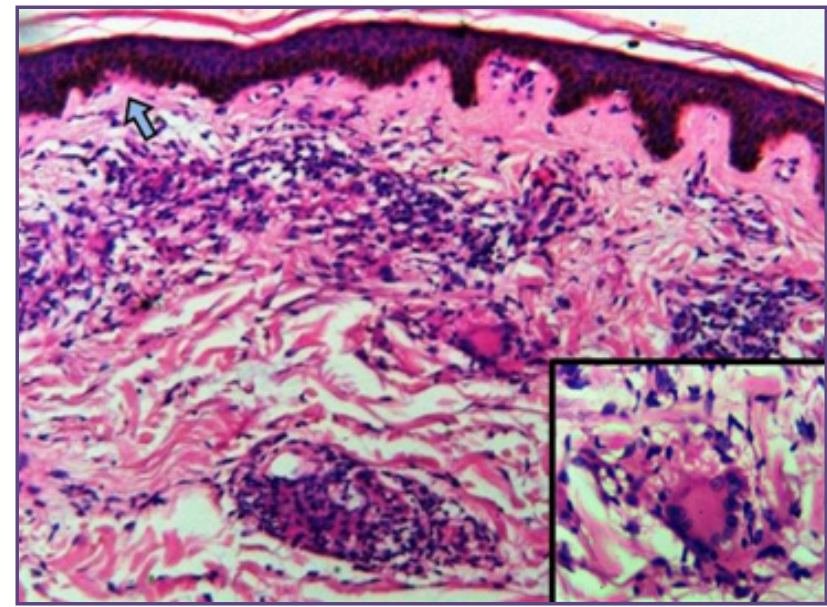

Fig. 2: Microscopic picture of polar tuberculoid leprosy with well-formed epithelioid cell granulomas in the dermis, surrounded by dense lymphocytic infiltration extending into the basal layer (arrow) (H and E, 10x). Inset shows a well-formed epithelioid cell granuloma with giant cells (Hematoxylin and Eosin, 40x).

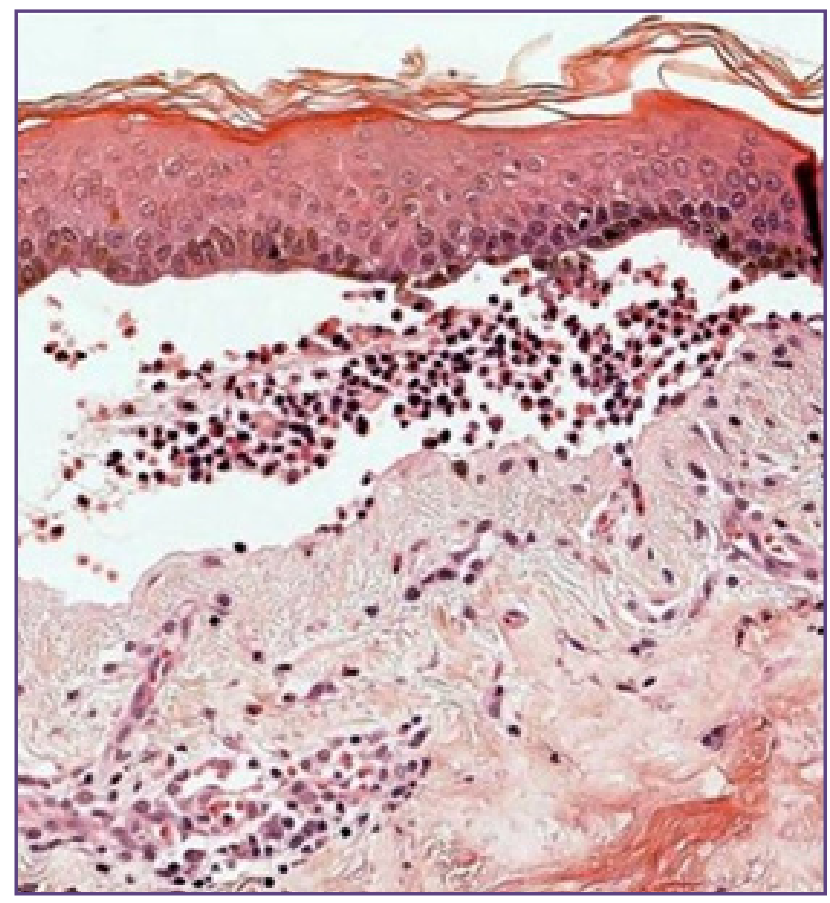

Fig. 4: Microscopic picture of bullous pemphigoid showing epidermis with subepidermal bulla filled with plasma and moderate infiltration of neutrophils and eosinophils. (Hematoxylin and Eosin, 40x).

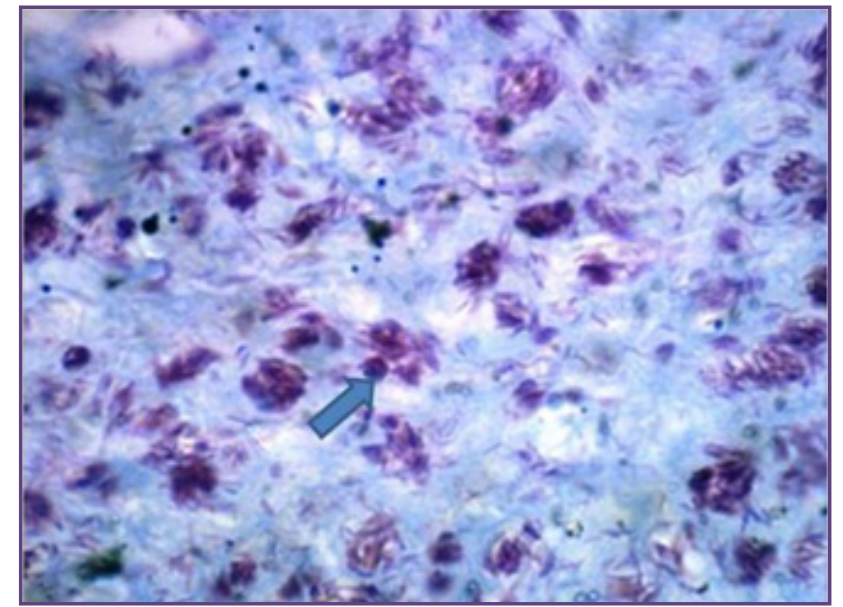

Fig. 3: Microscopic picture of lepromatous leprosy showing positive Fite-Faraco stain for lepra bacilli; bacterial index 6+; globi seen (arrow), (Fite-Faraco,100x).

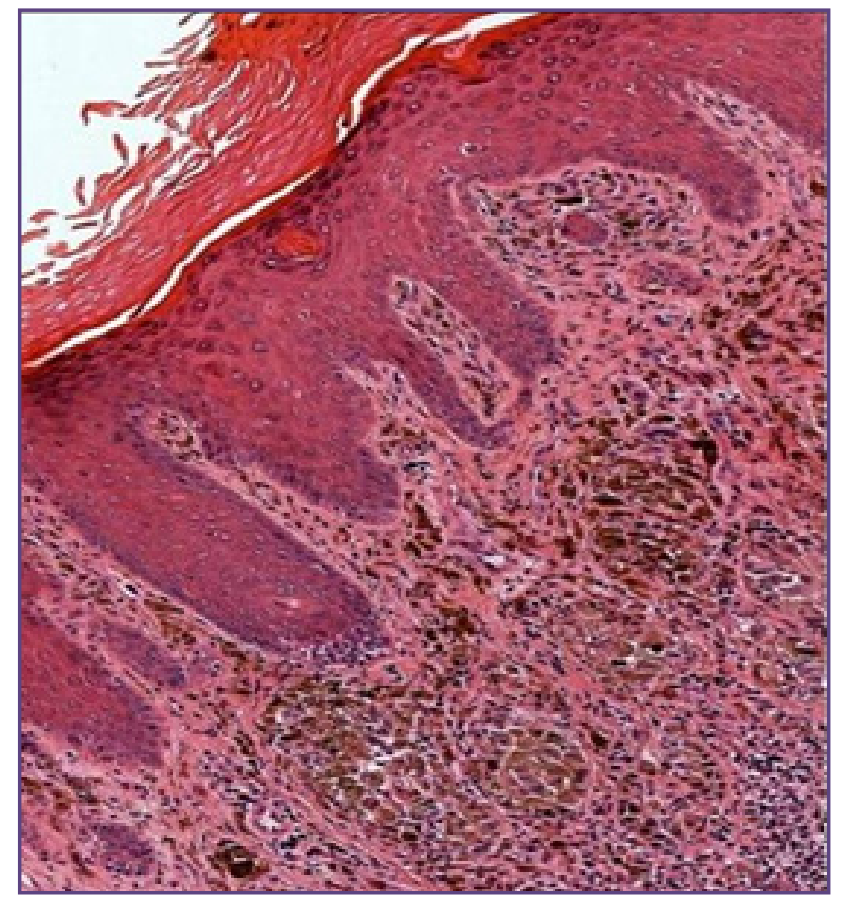

Fig. 5: Microscopic picture of malignant melanoma showing a broad proliferation of large atypical melanocytes arranged in poorly formed nests at the dermoepidermal junction.(Hematoxylin and Eosin, 40x). 


\section{Discussion}

The spectrum of dermatological lesions varies significantly from region to region due to social, cultural, economic and environmental factors. In this study, skin lesions were found to occur in all age groups, but the highest incidence was seen in the age group of 21 to 30 years (18.6\%), which is in concordance with the observations of previous researchers. ${ }^{6,8,9}$ However, a cross-sectional study on skin diseases by a study noted that the most common age group involved was 11 to 20 years with 164 (31.4\%) cases. ${ }^{10}$ This observation might be due to the crosssectional nature of the study and geographical domicile. Skin lesions were more prevalent in males (51\%). Similar observations were made by other authors with $56 \%$, $56.7 \%$ and $56.9 \%$, respectively. ${ }^{4,6,11}$ However, a very high incidence of dermatological lesions (91.2\%) was found in females in another study. ${ }^{8}$ The reasons attributed are the socioeconomic profile and nature of lifestyle.

There was specific histopathological diagnosis found in $87.9 \%$ of cases. The rate of inconclusive diagnosis is acceptable upto $20 \%$ in a given point of time beyond which quality control measures needs to be addressed. ${ }^{2}$ Majority of the observed skin lesions were inflammatory lesions $(59.5 \%)$ followed by skin neoplasms $(22.4 \%)$. The most frequently encountered inflammatory conditions were infective granulomatous lesions the predominant lesion being leprosy (9.6\%). A five year retrospective study conducted on 1280 skin biopsies found that granulomatous skin lesions constituted $24.4 \%$ of all lesions, which was similar to this study. ${ }^{12}$

Granulomatous skin lesions were more common in males $(62.5 \%)$ with male to female ratio of 1.67:1. A similar observation was made by previous researchers. ${ }^{12,13}$ The reasons proposed for the high incidence of granulomatous skin lesions among males is due to increased exposure to infectious agents owing to environmental conditions, poor quality of life and altered immune status. ${ }^{13}$ In this study, 22.5 $\%$ of all granulomatous skin lesions were seen in the age group of 21 to 30 years. A study conducted in West Bengal found that the majority of the patients with granulomatous skin lesions belonged to the age group between 11 to 20 years, whereas another study observed that majority of the granulomatous lesions occur in 41 to 50 years of age. ${ }^{12,13}$ The reason attributed for the non-concordance is the varied states of immune response to noxious stimuli triggering the lesions. Among the granulomatous lesions studied, leprosy $(64.2 \%)$ was the most frequent disease, followed by cutaneous tuberculosis $(19.2 \%)$. The Borderline tuberculoid leprosy constituted $44.1 \%$ and lepromatous leprosy constituted $10 \%$ cases. Similar observations were noted in earlier studies conducted by other authors. ${ }^{12,13} \mathrm{Few}$ cases of classical histoid leprosy showed positive FiteFaraco stain for lepra bacilli (Figure 3).

Infectious skin lesions accounted for $15.1 \%$ of the total skin lesions studied. Majority of the infectious skin lesions were of bacterial etiology ( $81 \%$ ) followed by viral etiology in $14 \%$ cases. This finding was similar to a study conducted in Puducherry which reported infectious skin diseases in $12 \%$ of cases,, whereas another study carried out in Madhya Pradesh documented that $53.6 \%$ of patients had infectious dermatoses, among which the majority comprised of bacterial dermatosis. ${ }^{8,14}$

Papulosquamous skin lesions were seen in a total of 118 cases (14.8\%) among which lichen planus was the most predominant lesion noted in 43 cases $(5.4 \%)$ followed by psoriasis in 34 cases(4.3\%). This finding was coincident with the findings of other studies which also observed lichen planus as the most common papulosquamous lesion. ${ }^{6,9}$ However, few studies have highlighted psoriasis as the most common lesion, followed by lichen planus. ${ }^{8,15}$ Among cutaneous vasculitis, the majority of the cases were leucocytoclastic vasculitis which correlated well with the observations of a similar study. ${ }^{8}$ However, the antibody titres and interpretation was not done as the histopathological findings were classical and striking on light microscopy.

Vesicobullous lesions were observed in a total of 38 cases which accounted for $4.8 \%$ of all skin lesions. Pemphigus vulgaris and bullous pemphigoid were the most common vesiculobullous skin lesions followed by pemphigus foliaceus and dermatitis herpetiformis. These findings were comparable to the findings of another study which documented $2.7 \%$ of dermatoses were vesicobullous lesions, among which pemphigus vulgaris was the most predominant lesion followed by bullous pemphigoid and pemphigus foliaceus. ${ }^{6}$ The only acceptable limitation with regard to vesiculobullous lesions was the lack of immunofluorescent study for confirmation. Connective tissue disorders were found in $5.5 \%$ of the skin lesions, among which morphea was the predominant lesion which is similiar to previous studies. ${ }^{8,14}$

Neoplastic skin lesions constituted a total of 192 cases (22.4\%) of which 122 lesions (63.5\%) were benign and 70 cases $(36.5 \%)$ were malignant lesions. Keratinocytic tumors were the most commonly encountered neoplastic lesions in both benign and malignant skin tumors, respectively. Among the malignant skin tumors, squamous cell carcinoma was the most prevalent cancerous lesion followed by basal cell carcinoma and malignant melanoma. These findings concurred with the findings in studies 
conducted by other authors. ${ }^{16,17}$ Another study conducted on skin tumors in Ahmedabad revealed keratinocytic tumors to be the most prevalent benign and malignant skin tumors, respectively with basal cell carcinoma as the most prevalent malignant skin tumor. ${ }^{18}$

Clinicopathological correlation was performed in the studied cases to analyze the causes for non-specific reports and to evaluate the level of concordance with clinical and histopathological diagnosis. The concordance was noted among 430 cases (53.8\%). A clinicopathological correlation of $43.9 \%$ was observed in a similar study. ${ }^{6}$ However, a higher clinicopathological correlation of $85.8 \%$ was observed in a single study. ${ }^{19}$ The reason attributed for the higher clinicopathological correlation is the classical clinical presentation of lesions. Many cases which had nonclassical clinical presentations led to clinical differential diagnoses, thereby retarding the clinicopathological correlation concordance rate.

Melanocytic tumors had good clinicopathological correlation (benign $83.3 \%$ and malignant 100\%), which was statistically significant, whereas tumors with follicular differentiation had very poor clinicopathological correlation due to their atypical presentation in terms of sites and nature of the lesions. This can be due to the fact that follicular, eccrine or apocrine differentiation of a tumor can only be determined based on histopathological appearance and not on clinical presentation. A single case of histopathologically proven basal cell carcinoma was clinically diagnosed as malignant melanoma. Similar case reports have been documented by other authors since the pigmented and nodular variants of basal cell carcinoma could clinically imitate malignant melanocytic tumors. ${ }^{20,21}$

Infectious and granulomatous skin lesions showed good clinicopathological correlation which was statistically significant. All histopathologically proven cases of leprosy (Hansen's disease) were at par with clinical diagnosis in the present study.

Among the papulosquamous skin lesions, psoriasis, lichen planus and pityriasis rubra pilaris showed good clinicopathological correlation. A discordance of $14.7 \%$ was seen in histopathologically proven cases of psoriasis owing to it being clinically diagnosed as lichen planus and mycosis fungoides, respectively in few instances due to the similar clinical presentations. There have been case reports of psoriasis mimicking mycosis fungoides, a primary cutaneous lymphoma documented by other authors. ${ }^{22-24}$ Few cases of histolopathologically proven lichen planus were clinically diagnosed as pityriasis rosea. A similar case report of lichen planus being mimicked by pityriasis rosea is available in literature. ${ }^{25}$ It is often challenging to differentiate papulosquamous lesions from one another based on their clinical presentations and a meticulous histopathological examination is essential to distinguish between lesions.

A histopathologically proven case of lupus erythematosus was clinically diagnosed as lupus vulgaris in the present study. A similar case report of lupus erythematosis mimicking lupus vulgaris was also found in world literature. ${ }^{26}$

Thus, a high level of clinicopathological correlation can be achieved by providing the sufficient clinical descriptive knowledge, thereby increasing the probability of making an accurate diagnosis. It was also helpful in instances where non-conformity was encountered. Large multicentre histopathological studies are recommended to assess the pattern of skin diseases based on histomorphological evaluation to develop clinicopathological correlation and public health strategies towards improving patient care.

\section{Conclusion}

The prevalence of dermatological lesions in the study population was higher in the third to sixth decades of life with a male preponderance. Granulomatous inflammation is still rampant with leprosy and tuberculosis as the leading causes in spite of extensive programmes and preventive measures. Infectious inflammatory dermatoses such as leprosy and neoplastic lesions such as malignant melanocytic tumors showed good clinicopathological correlation. In cases where skin biopsy delivered a nonspecific diagnosis, infective or neoplastic etiology was ruled out. The heterogeneity in the clinical presentation of skin diseases makes histopathological examination a gold standard technique for final diagnosis and clinicopathological correlation.

\section{References}

1. Singh S, Debnath A, Datta D, Chakravarty S, Chaubey RN. Histopathological Evaluation of Skin Lesions with Special Reference to Skin Adnexal Tumors in a Tertiary Centre of North-Eastern India-A Three Year Study. IOSR-JDMS. 2016;15:34-9.

2. Sarkar SK, Islam A, Sen KG, Ahmed ARS. Pattern of skin diseases in patients attending OPD of Dermatology Department at Faridpur Medical College Hospital, Bangladesh. Faridpur Med Coll J. 2010;5:14-16.

3. World Health Organization. Epidemiology and management of common skin diseases in children in developing countries. Geneva: World Health Organization; 2005.

4. Mehar R, Jain R, Kulkarni C, Narang S, Mittal M, Patidar H. Histopathological study of dermatological lesions A retrospective approach. Int $\mathrm{J}$ Med Sci Public Health. 2014;3:1082-85.

5. Sharma S, Trivedi DP, Vyas R. Evaluation of Epidermal Reaction Pattern and Assessment of Histopathological 
Findings of Various Skin Disorders. J Contemp Med Res. 2016;3:1755-59.

6. Narang S, Jain R. An evaluation of histopathological findings of skin biopsies in various skin disorders. Ann Pathol Lab Med. 2015;2:A42-A46.

7. Aslan C, Gktay F, Mansur AT, Aydngz kbal E, Gne P, Ekmeki TR. Clinicopathological consistency in skin disorders: A retrospective study of 3949 pathological reports. J Am Acad Dermatol. 2012;66:393-400.

8. Gulia SP, Wadhai SA, Lavanya M, Menon R, Chaudhary M, Kumar SP. Histopathological pattern of skin diseases in a teaching hospital Puducherry. Int J Recent Trend Sci Technol. 2014;11:45-50.

9. Veldurthy V, Shanmugam C, Sudhir N, Sirisha O, Motupalli C, Rao N, et al. Pathological study of non-neoplastic skin lesions by punch biopsy. Int J Res Med Sci. 2015;1985-88.

10. Grover S, Ranyal R, Bedi M. A cross section of skin diseases in rural Allahabad. Indian J Dermatol. 2008;53:179-181.

11. Agarwal D, Singh K, Saluja SK, Kundu PR, Kamra H, Agarwal R. Histopathological Review of Dermatological Disorders with a Keynote to Granulomatous Lesions: A Retrospective Study. Int J Sci Stud 2015;3:66-69.

12. Chakrabarti S, Pal S, Biswas BK, Bose K, Pal S, Pathak S. Clinico-pathological study of cutaneous granulomatous lesions-a $5 \mathrm{yr}$ experience in a tertiary care hospital in India. Iran J Pathol. 2016;11:54-60.

13. Gautam K, Pai RR, Bhat S. Granulomatous lesions of the skin. J Pathol Nepal. 2011;1:81-86.

14. Goyal N, Jain P, Malik R, Koshti A. Spectrum of non neoplastic skin diseases: A histopathology based clinicopathological correlation study. Sch J App Med Sci. 2015;3:444-449.

15. Chichani S, Negi SR, Kalla AR, Gaur S. Study of histopathology of papulosquamous lesion of skin a prospective and retrospective study. IJAR. 2016;2:115117
16. Achalkar GV. Clinicopathological evaluation of nonneoplastic and neoplastic skin lesions: A study of 100 cases. Indian J Pathol Oncol 2019;6:118-122.

17. Gaikwad SL, Kumawat UD, Sakhare NA, D'costa GF. Histopathological spectrum of skin lesions - Experience at rural based hospital. International $\mathrm{J}$ of Current Research 2016; 8:36223-27.

18. Patel N, Suthar T, Suthar H, Arora A. A study of tumours, tumour like lesions and cysts of epidermis and its appendages. Indian J Clin Exp Dermatol. 2018;4:194-200.

19. Gupta P, Karuna V, Grover K. The histopathological spectrum of skin diseases with emphasis on clinicopathological correlation: A prospective study. IP J Diagn Pathol Oncol. 2018;3:91-95.

20. Petrescu IR, Sajin M, Costache M, Simion G. Basal cell carcinoma miming cutaneous melanoma. Rom J Morphol Embryol. 2011;52:197-201.

21. Hasbún Acuña P, Cullen Aravena R, Maturana Donaire C, Ares Mora R, Porras Kusmanic N. Pigmented basal cell carcinoma mimicking a superficial spreading melanoma. Medwave. 2016 Dec 20; 16:e6805.

22. Vaudreuil AM, Stroud CM, Hsu S. Psoriasis mimicking mycosis fungoides clinically. Dermatol Online J. 2017 May 15;23.

23. Doukaki S, Aricò M, Bongiorno MR. A Rare Presentation of Mycosis Fungoides Mimicking Psoriasis Vulgaris. Case Rep Dermatol. 2009;1:60-5.

24. Elmer KB, George RM. Cutaneous T-Cell Lymphoma Presenting as Benign Dermatoses. Am Fam Physician. 1999;59:2809.

25. Marshall J. Pityriasis rosea; a review of its clinical aspects and a discussion of its relationship to pityriasis lichenoides et varioliformis acuta and parapsoriasis guttata. S Afr Med J. 1956;30:210-8

26. Verma P, Pathania S, Kubba A. A rare case of unilateral discoid lupus erythematosus mimicking lupus vulgaris. BMJ Case Rep.2017;2017:bcr-2017-222218.

*Corresponding author:

Dr. Sowmya S, Professor and Head of Department of Pathology, Mahatma Gandhi Medical College and Research Institute, Sri Balaji Vidyapeeth University,Pillaiyarkuppam, Puducherry- 607402, India.

Email: drssowmya@hotmail.com

Financial or other Competing Interests: None. 